\title{
Patterns of spatial variability of seagrass epiphytes in the north-west Mediterranean Sea
}

David Balata $\cdot$ Ugo Nesti · Luigi Piazzi •

Francesco Cinelli

Published online: 18 April 2007

(C) Springer-Verlag 2007

Erratum to: Mar Biol

DOI 10.1007/s00227-006-0559-y

Unfortunately Tables 1-5 contained errors. The correct tables $1-5$ are shown below.

The online version of the original article can be found at http://dx.doi.org/10.1007/s00227-006-0559-y.

D. Balata $(\bowtie)$

University of Pisa, Pisa, Italy

e-mail: dbalata@biologia.unipi.it

U. Nesti

I.C.R.A.M., Via di Casalotti 300, Rome 00166, Italy

L. Piazzi · F. Cinelli

Dipartimento di Biologia, Università di Pisa,

Via Volta 6, Pisa 57126 Italy 
Table 1 List of taxa identified in the epiphytic assemblages of leaves and rhizomes

\begin{tabular}{lll}
\hline Taxa & Leaves & Rhizomes
\end{tabular}

\section{Chlorophyta}

Caulerpa racemosa (Forsskål) C. Agardh var. cylindracea (Sonder) Verlaque, Huisman et Boudouresque

Chaetomorpha linum (O. F. Müller) Kützing

Cladophora prolifera (Roth) Kützing

Derbesia tenuissima (Moris et De Notaris) P. L. et H. M. Crouan

Flabellia petiolata (Turra) Nizamuddin

Pseudochlorodesmis furcellata (Zanardini) Børgesen

Siphonocladus pusillus (C. Agardh ex Kützing) Hauck

\section{Phaeophyta}

Asperococcus sp.

Cladosiphon cylindricus (Sauvageau) Kylin

Cladosiphon irregularis (Sauvageau) Kylin

Dictyota dichotoma (Hudson) J. V. Lamouroux

Dictyota fasciola (Roth) J. V. Lamouroux

Dictyota linearis (C. Agardh) Greville

Ectocarpus siliculosus (Dillwyn) Lyngbye

Elachista intermedia P. L. et H. M. Crouan

Giraudia sphacelarioides Derbés et Solier

Padina pavonica (Linnaeus) Thivy

Sphacelaria cirrosa (Roth) C. Agardh

\section{Rhodophyta}

Acrothamnion preissii (Sonder) Wollaston

Amphiroa rigida J. V. Lamouroux

Anthithamnion cruciatum (C. Agardh) Nägeli

Apoglossum ruscifolium (Turner) J. Agardh

Botryocladia boergesenii J. Feldmann

Botryocladia botryoides (Wulfen) J. Feldmann

Ceramium codii (Richards) G. Feldmann

Ceramium diaphanum (Lighfoot) Roth

Chondria mairei $\mathrm{G}$. Feldmann

Contarinia peyssonelliaeformis Zanardini

Dipterosiphonia dendritica (C. Agardh) F. Schmitz

Dipterosiphonia rigens (Schousboe) Falkenberg

Feldmannophycus rayssiae (Feldmann et Feldmann-Mazoyer) Augier et Boudouresque

Haliptilon virgatum (Zanardini) Garbary et H. W. Johansen

Heterosiphonia crispella (C. Agardh) Wynne

Hydrolithon cruciatum (Bressan) Chamberlain

Hydrolithon farinosum (Lamouroux) Penrose et Chamberlain

Hypoglossum hypoglossoides (Stackhouse) Collins et Harvey

Jania rubens J. V. Lamouroux

Laurencia spp.

Lithophyllum cystoseirae (Hauck) Heydrich

Mesophyllum lichenoides (J. Ellis) M. Lemoine

Monosporus pedicellatus (Smith) Solier

Nitophyllum punctatum (Stackhouse) Greville

Peyssonnelia bornetii Boudouresque et Denizot

Plocamium cartilagineum (Linnaeus) Dixon

$\begin{array}{ll}- & + \\ + & - \\ + & + \\ - & + \\ - & + \\ + & + \\ + & +\end{array}$

$\begin{array}{ll}+ & + \\ + & + \\ + & - \\ + & + \\ + & + \\ - & + \\ + & - \\ + & + \\ - & + \\ - & + \\ - & + \\ - & + \\ - & + \\ - & - \\ + & + \\ + & - \\ + & + \\ + & + \\ + & + \\ + & + \\ - & + \\ - & + \\ + & + \\ + & + \\ - & + \\ - & + \\ & +\end{array}$


Table 1 continued

\begin{tabular}{l} 
Taxa \\
\hline Pneophyllum coronatum (Rosanoff) Penrose \\
Pneophyllum fragile Kützing \\
Polysiphonia spp. \\
Rhodymenia sp. \\
Womersleyella setacea (Hollenberg) R. E. Norris \\
Wrangelia penicillata $($ C. Agardh) C. Agardh
\end{tabular}

Rhizopoda

Cyclocibicides vermiculatus d'Orbigny

Elphidium crispum Linnaeus

Lobatula lobatula Walker et Jacob

Miniacina miniacea Linnaeus

Planorbulina mediterranensis Walker et Jacob

Rosalina brady Cushman

\section{Porifera}

Clathrina contorta Bow

Cliona viridis $\mathrm{Schmidt}$

Dysidea sp.

Oscarella lobularis Schmidt

Plakortis simplex Schulze

Sycon raphanus $\mathrm{Schmidt}$

Tethya citrina Sarà et Melone

\section{Cnidaria}

Laomedea angulata Hinks

Dynamena sp.

Obelia geniculata Linnaeus

Orthopyxis asymmetrica Stechow

Plumularia obliqua Thompson

Podocoryne carnea M. Sars

Sertularia perpusilla Stechow

\section{Anellida}

Janua pagenstecheri Quatrefageau

Pomatoceros triqueter Linnaeus

\section{Bryozoa}

Aetea truncata Landsborough

Beania hirtissima Heller

Beania magellanica Bush

Caberea boryi Audouin

Callopora lineata Linnaeus

Carbasea papyrea Pallas

Cellepora pumicosa Pallas

Cellaria salicornioides Lamouroux

Chlidonia pyriformis Bertolini

Chorizopora brogliartii Audouin

Collarina balzaci Audouin et Savigny

Cribrilaria radiata Moll

Cryptosula pallasiana Moll

Electrae posidonia Gautier

Escharoides coccinea Abildgaard
Leaves

Rhizomes

$+$

$+$

$+$

$-$

$+$

$+$

$+$

$+$

$+$

-

$+$

$+$

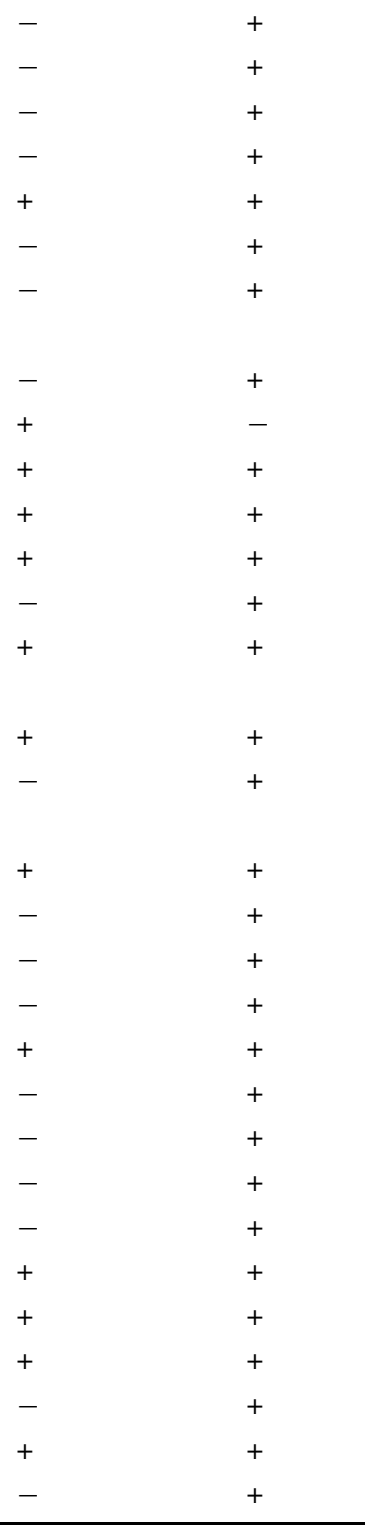


Table 1 continued

\begin{tabular}{llc}
\hline Taxa & Leaves & Rhizomes \\
\hline Fenestrulina malusii Audouin & + & + \\
Idmonea sp. & + & + \\
Lichenopora radiata Audouin et Savigny & + & + \\
Margaretta cereoides Ellis et Solander & - & + \\
Microporella ciliata Pallas & + & + \\
Pherusella tubulosa Ellis et Solander & - & + \\
Plagioecia patina Lamarck & - & + \\
Reteporella sp. & - & + \\
Schizobrachiella sanguinea Norman & + & + \\
Scrupocellaria reptans Linneaus & + & + \\
Tubulipora sp. & & + \\
Tunicata & + & + \\
Botryllus schlosseri Pallas & - & + \\
Didemnum fulgens Milne-Edwards & - & + \\
Diplosoma listerianum Milne-Edwards & - & + \\
Sidnyum turbinatum Savigny & + & \\
Trididemnum cereum Giard & & + \\
\hline
\end{tabular}

Table 2 PERMANOVA on the structure of the epiphytic assemblages of leaves and of rhizomes

\begin{tabular}{|c|c|c|c|c|c|c|c|c|c|c|c|}
\hline \multirow[b]{2}{*}{ Source } & \multirow[b]{2}{*}{ df } & \multicolumn{4}{|c|}{ Epiphytic assemblages of leaves } & \multicolumn{4}{|c|}{ Epiphytic assemblages of rhizomes } & \multirow[b]{2}{*}{$\begin{array}{l}\text { Denominator } \\
\text { for } F\end{array}$} & \multirow[b]{2}{*}{$\begin{array}{l}\text { Number of } \\
\text { permutable } \\
\text { units }\end{array}$} \\
\hline & & MS & Pseudo F & $\mathbf{P}$ & $\mathbf{P}(\mathbf{M C})$ & MS & Pseudo F & $\mathbf{P}$ & $\mathbf{P}(\mathbf{M C})$ & & \\
\hline Habitat $=\mathrm{Ha}$ & 2 & 66453.63 & 0.79 & 0.574 & 0.605 & 23443.40 & 0.70 & 0.607 & 0.806 & $\mathrm{Me}(\mathrm{Ha})$ & 6 \\
\hline Meadow $(\mathrm{Ha})=\mathrm{Me}(\mathrm{Ha})$ & 3 & 84027.47 & 36.50 & 0.002 & 0.001 & 33658.71 & 5.19 & 0.001 & 0.001 & $\mathrm{Si}(\mathrm{Me}(\mathrm{Ha}))$ & 12 \\
\hline Site $(\mathrm{Me}(\mathrm{Ha}))=\mathrm{Si}(\mathrm{Me}(\mathrm{Ha}))$ & 6 & 2302.18 & 1.79 & 0.046 & 0.032 & 6480.13 & 1.01 & 0.439 & 0.447 & $\mathrm{Pl}(\mathrm{Si}(\mathrm{Me}(\mathrm{Ha}))$ & 36 \\
\hline $\begin{array}{l}\text { Plot }(\mathrm{Si}(\mathrm{Me}(\mathrm{Ha})))= \\
\quad \operatorname{Pl}(\mathrm{Si}(\mathrm{Me}(\mathrm{Ha})))\end{array}$ & 24 & 1283.57 & 1.74 & 0.001 & 0.001 & 6395.89 & 4.00 & 0.001 & 0.001 & Residual & 180 \\
\hline Residual & 144 & 736.48 & & & & 1597.37 & & & & & \\
\hline Total & 179 & & & & & & & & & & \\
\hline
\end{tabular}

Significant effects are indicated in bold 
Table 3 Results of the indicator species analysis (IndVal), of epyphytic assemblages of leaves

\begin{tabular}{|c|c|c|c|c|c|c|c|c|c|}
\hline \multirow[b]{2}{*}{ Meadows } & \multirow[b]{2}{*}{ Species } & \multirow[b]{2}{*}{ IndVal } & \multirow[b]{2}{*}{ Level } & \multicolumn{6}{|c|}{ Meadows } \\
\hline & & & & $\begin{array}{l}L \\
a / p\end{array}$ & $\begin{array}{l}B \\
a / p\end{array}$ & $\begin{array}{l}V \\
a / p\end{array}$ & $\begin{array}{l}M \\
a / p\end{array}$ & $\begin{array}{l}G \\
a / p\end{array}$ & $\begin{array}{l}P \\
a / p\end{array}$ \\
\hline \multirow[t]{4}{*}{$L$} & Pneuphyllum spp. & 84.40 & 1 & $104 . / 30$ & $114 . / 30$ & $0 . / 3$ & $87 . / 30$ & $111 . / 30$ & $141 . / 29$ \\
\hline & Womersleyella setacea & 51.98 & 1 & 3./17 & $0 . / 0$ & $0 . / 0$ & $0 . / 0$ & $0 . / 3$ & $0 . / 0$ \\
\hline & Electrae posidoniae & 49.83 & 1 & $32 . / 25$ & $0 . / 0$ & $0 . / 4$ & $21 . / 26$ & $0 . / 10$ & $0 . / 4$ \\
\hline & Rosalina brady & 21.86 & 1 & $1 . / 9$ & $0 . / 1$ & $0 . / 0$ & $0 . / 0$ & $0 . / 3$ & $0 . / 1$ \\
\hline \multirow[t]{4}{*}{$B$} & Laurencia spp. & 64.01 & 1 & $0 . / 0$ & $9 . / 24$ & $1 . / 9$ & $1 . / 7$ & $0 . / 0$ & $0 . / 0$ \\
\hline & Hypoglossum hypoglossoides & 30.87 & 1 & $0 . / 0$ & 1./18 & $1 . / 15$ & $0 . / 0$ & $0 . / 4$ & $0 . / 2$ \\
\hline & Collapora lineata & 13.64 & 3 & $0 . / 0$ & $0 . / 5$ & $0 . / 1$ & $0 . / 0$ & $0 . / 0$ & $0 . / 0$ \\
\hline & Ectocarpus siliculosus & 11.45 & 15 & $0 . / 0$ & $1 . / 8$ & $0 . / 1$ & $0 . / 0$ & $0 . / 0$ & $1 . / 2$ \\
\hline \multirow[t]{5}{*}{$V$} & Plumularia obliqua & 100 & 1 & $0 . / 0$ & $0 . / 0$ & $106 / 30$ & $0 . / 0$ & $0 . / 0$ & $0 . / 0$ \\
\hline & Heterosiphonia crispella & 79.61 & 1 & $0 . / 1$ & $0 . / 1$ & 24./30 & $2 . / 14$ & $1 . / 5$ & $3 . / 13$ \\
\hline & Trididemnum cereum & 48.3 & 1 & $0 . / 0$ & $0 . / 0$ & $1 . / 16$ & $0 . / 1$ & $0 . / 0$ & $0 . / 1$ \\
\hline & Obelia geniculata & 46.77 & 1 & $0 . / 0$ & $1 . / 9$ & $4 . / 20$ & $0 . / 0$ & $0 . / 0$ & $1 . / 8$ \\
\hline & Cladosiphon irregularis & 33.83 & 1 & $0 . / 0$ & $1 . / 3$ & $1 . / 14$ & $0 . / 0$ & $0 . / 0$ & $0 . / 0$ \\
\hline \multirow[t]{2}{*}{$M$} & Dictyota linearis & 60.35 & 1 & $4 . / 11$ & $4 . / 10$ & $0 . / 2$ & $33 . / 23$ & $0 . / 3$ & $0 . / 2$ \\
\hline & Wrangelia penicillata & 53.16 & 1 & $1 . / 3$ & $0 . / 0$ & $0 . / 16$ & $1 . / 28$ & $0 . / 0$ & $0 . / 0$ \\
\hline \multirow[t]{5}{*}{$P$} & Sphacelaria cirrosa & 71.97 & 1 & $0 . / 0$ & $1 . / 27$ & $0 . / 0$ & $0 . / 0$ & $9 . / 27$ & $1 . / 19$ \\
\hline & Tubulipora sp. & 42.35 & 1 & $0 . / 0$ & $5 . / 28$ & $0 . / 1$ & $0 . / 0$ & $7 . / 24$ & $1 . / 7$ \\
\hline & Polysiphonia spp. & 32.64 & 1 & $0 . / 1$ & $0 . / 0$ & $0 . / 0$ & $0 . / 0$ & $1 . / 11$ & $0 . / 0$ \\
\hline & Ceramium codii & 24 & 1 & $0 . / 0$ & $0 . / 0$ & $0 . / 0$ & $0 . / 0$ & $0 . / 9$ & $0 . / 4$ \\
\hline & Ceramium diaphanum & 19.1 & 2 & $0 . / 3$ & $0 . / 0$ & $0 . / 0$ & $0 . / 0$ & $1 . / 7$ & $0 . / 1$ \\
\hline \multirow[t]{3}{*}{$G$} & Elachista intermedia & 64.05 & 1 & $0 . / 0$ & $1 . / 13$ & $8 . / 23$ & $0 . / 0$ & $19 . / 27$ & $54 . / 29$ \\
\hline & Elphidium crispum & 18.75 & 1 & $0 . / 0$ & $1 . / 8$ & $0 . / 1$ & 0 & $1 . / 3$ & $2 . / 9$ \\
\hline & Chorizopora brogniartii & 9.58 & 20 & $0 . / 0$ & $0 . / 2$ & $0 . / 0$ & $0 . / 0$ & $0 . / 2$ & $1 . / 4$ \\
\hline
\end{tabular}


Table 4 Results of the indicator species analysis (IndVal), of epyphytic assemblages of rhizomes
Numbers indicate cases (numbers of samples out of 30) where each taxon is significantly different in abundance $a$ or presence $p$ among the studied meadows. $L$ Livorno, $B$ Baratti, $V$ Secche di Vada, $M$ Secche della Meloria, $G$ Isola di Gorgona, $P$ Isola di Pianosa

\begin{tabular}{|c|c|c|c|c|c|c|c|c|c|}
\hline \multirow[b]{2}{*}{ Meadows } & \multirow[b]{2}{*}{ Species } & \multirow[b]{2}{*}{ IndVal } & \multirow[b]{2}{*}{ Level } & \multicolumn{6}{|c|}{ Meadows } \\
\hline & & & & $\begin{array}{l}L \\
a / p\end{array}$ & $\begin{array}{l}B \\
a / p\end{array}$ & $\begin{array}{l}V \\
a / p\end{array}$ & $\begin{array}{l}M \\
a / p\end{array}$ & $\begin{array}{l}G \\
a / p\end{array}$ & $\begin{array}{l}P \\
a / p\end{array}$ \\
\hline \multirow[t]{4}{*}{$L$} & Sphacelaria cirrosa & 35.26 & 1 & 2./12 & $0 . / 0$ & $0 . / 1$ & $0 . / 0$ & $0 . / 0$ & $0 . / 1$ \\
\hline & Pseudochlorodesmis furcellata & 19.92 & 1 & 1./8 & $0 . / 1$ & $0 . / 1$ & $0 . / 0$ & $0 . / 0$ & $0 . / 0$ \\
\hline & Schizobrachiella sanguinea & 14.83 & 4 & $6 . / 7$ & $1 . / 2$ & $2 . / 6$ & $0 . / 1$ & $0 . / 0$ & $0 . / 0$ \\
\hline & Rhodymenia sp. & 13.77 & 1 & $1 . / 6$ & $0 . / 0$ & $0 . / 0$ & $0 . / 2$ & $0 . / 0$ & $0 . / 0$ \\
\hline \multirow[t]{2}{*}{$B$} & Plakortis simplex & 33.47 & 1 & $0 . / 3$ & $18 . / 19$ & $7 . / 6$ & $1 . / 2$ & $6 . / 4$ & $2 . / 2$ \\
\hline & Pherusella tubulosa & 24.61 & 1 & $0 . / 0$ & $5 . / 13$ & $1 . / 4$ & $0 . / 0$ & $2 . / 7$ & $1 . / 5$ \\
\hline \multirow[t]{7}{*}{$V$} & Fenestrulina malusii & 56.61 & 1 & $0 . / 0$ & $4 . / 19$ & $15 . / 30$ & $1 . / 2$ & $3 . / 9$ & $0 . / 0$ \\
\hline & Janua pagenstecheri & 51.44 & 1 & $0 . / 0$ & $0 . / 5$ & $2 . / 19$ & $0 . / 1$ & $0 . / 3$ & $0 . / 0$ \\
\hline & Cellepora pumicosa & 41.94 & 1 & $0 . / 0$ & $1 . / 4$ & 8./15 & $0 . / 0$ & $0 . / 0$ & $0 . / 0$ \\
\hline & Laomedea angulata & 36.10 & 1 & $0 . / 0$ & $10 . / 25$ & $14 . / 23$ & $1 . / 6$ & $0 . / 0$ & $0 . / 0$ \\
\hline & Sidnyum turbinatum & 34.07 & 1 & $1 . / 9$ & $2 . / 4$ & 7./18 & $2 . / 12$ & $0 . / 2$ & $1 . / 9$ \\
\hline & Cribrilaria radiata & 27.00 & 1 & $0 . / 0$ & $0 . / 1$ & $2 . / 13$ & $1 . / 6$ & $0 . / 0$ & $0 . / 0$ \\
\hline & Callopora lineata & 19.68 & 4 & $0 . / 3$ & $2 . / 12$ & $3 . / 12$ & $0 . / 1$ & $0 . / 0$ & $0 . / 3$ \\
\hline \multirow[t]{6}{*}{$M$} & Dictyota dichotoma & 68.47 & 1 & $3 . / 13$ & $8 . / 19$ & $0 . / 1$ & $43 . / 26$ & $0 . / 0$ & $3 . / 13$ \\
\hline & Didemnum fulgens & 45.07 & 1 & $0 . / 3$ & $0 . / 1$ & $1 . / 1$ & $35 . / 14$ & $0 . / 0$ & $0 . / 3$ \\
\hline & Margaretta cereoides & 42.31 & 1 & $0 . / 3$ & $0 . / 1$ & $2 . / 4$ & $12 . / 17$ & $1 . / 0$ & $0 . / 3$ \\
\hline & Dictyota linearis & 19.23 & 1 & $2 . / 7$ & $0 . / 0$ & $0 . / 0$ & $4 . / 8$ & $0 . / 0$ & $2 . / 7$ \\
\hline & Botryocladia boergesenii & 13.33 & 4 & $0 . / 0$ & $0 . / 0$ & $0 . / 0$ & $1 . / 4$ & $0 . / 0$ & $0 . / 0$ \\
\hline & Trididemnum cereum & 11.01 & 15 & $0 . / 0$ & $2 . / 2$ & $2 . / 4$ & $4 . / 6$ & $0 . / 0$ & $0 . / 0$ \\
\hline \multirow[t]{6}{*}{$G$} & Cliona viridis & 26.67 & 1 & $0 . / 0$ & $0 . / 0$ & $0 . / 0$ & $0 . / 0$ & 8./8 & $0 . / 0$ \\
\hline & Podocoryne carnea & 26.67 & 1 & $0 . / 0$ & $0 . / 0$ & $0 . / 0$ & $0 . / 0$ & $4 . / 8$ & $0 . / 0$ \\
\hline & Pneophyllum spp. & 23.02 & 2 & $1 . / 3$ & $0 . / 1$ & $1 . / 1$ & $1 . / 1$ & 10./11 & $3 . / 4$ \\
\hline & Hypoglossum hypoglossoides & 19.91 & 1 & $0 . / 3$ & $0 . / 0$ & $0 . / 0$ & $0 . / 0$ & 3./7 & $0 . / 1$ \\
\hline & Orthopyxis asymmetrica & 18.92 & 1 & $1 . / 7$ & $1 . / 2$ & $1 . / 4$ & $0 . / 2$ & 3./11 & $0 . / 0$ \\
\hline & Tubulipora sp. & 16.35 & 2 & $0 . / 0$ & $0 . / 1$ & $0 . / 0$ & $0 . / 0$ & $3 . / 7$ & $1 . / 6$ \\
\hline \multirow[t]{5}{*}{$P$} & Hydrolithon farinosum & 37.34 & 1 & $0 . / 0$ & $1 . / 1$ & $25 . / 20$ & $11 . / 10$ & $6 . / 10$ & $55 . / 20$ \\
\hline & Miniacina miniacea & 36.29 & 1 & $0 . / 0$ & $0 . / 1$ & $6 . / 13$ & $0 . / 0$ & $15 . / 22$ & $14 . / 27$ \\
\hline & Idmonea sp. & 33.71 & 1 & $0 . / 0$ & $4 . / 13$ & $0 . / 1$ & $0 . / 0$ & $3 . / 11$ & $5 . / 22$ \\
\hline & Peyssonnelia bornetii & 20.68 & 1 & $1 . / 5$ & $0 . / 0$ & $0 . / 0$ & $0 . / 0$ & $0 . / 0$ & $9 . / 7$ \\
\hline & Oscarella lobularis & 12.63 & 4 & $0 . / 1$ & $0 . / 0$ & $0 . / 0$ & $0 . / 0$ & $4 . / 2$ & $15 . / 5$ \\
\hline
\end{tabular}


Table 5 Results of four-way ANOVA on number of species and on total percentage cover of epiphytic assemblages of leaves and of rhizomes

\begin{tabular}{|c|c|c|c|c|c|c|c|c|c|c|c|c|c|}
\hline \multirow[b]{3}{*}{ Source } & \multirow[b]{3}{*}{ df } & \multicolumn{6}{|c|}{ Epiphytic assemblages of leaves } & \multicolumn{6}{|c|}{ Epiphytic assemblages of rhizomes } \\
\hline & & \multicolumn{3}{|c|}{ Number of species } & \multicolumn{3}{|c|}{ Percentage cover } & \multicolumn{3}{|c|}{ Number of species } & \multicolumn{3}{|c|}{ Percentage cover } \\
\hline & & MS & $\mathbf{F}$ & $\mathbf{P}$ & MS & $\mathbf{F}$ & $\mathbf{P}$ & MS & $\mathbf{F}$ & $\mathbf{P}$ & MS & $\mathbf{F}$ & $\mathbf{P}$ \\
\hline Habitat $=\mathrm{Ha}$ & 2 & 13.12 & 0.060 & NS & 30.93 & 29.400 & $*$ & 51.45 & 0.490 & NS & 296.13 & 0.470 & NS \\
\hline Meadow $(\mathrm{Ha})=\mathrm{Me}(\mathrm{Ha})$ & 3 & 222.57 & 4.460 & NS & 1.05 & 0.080 & NS & 105.69 & 1.110 & NS & 623.51 & 5.600 & $*$ \\
\hline Site $(\mathrm{Me}(\mathrm{Ha}))=\mathrm{Si}(\mathrm{Me}(\mathrm{Ha}))$ & 6 & 49.86 & 8.390 & $* * *$ & 12.81 & 1.570 & NS & 95.38 & 4.560 & $* *$ & 111.26 & 1.030 & NS \\
\hline Plot $(\mathrm{Si}(\mathrm{Me}(\mathrm{Ha})))=\operatorname{Pl}(\mathrm{Si}(\mathrm{Me}(\mathrm{Ha})))$ & 24 & 5.94 & 1.760 & $* *$ & 8.17 & 2.120 & $* *$ & 20.91 & 2.080 & $* *$ & 107.58 & 4.630 & $* * *$ \\
\hline Residual $=$ Res & 144 & 3.37 & & & 3.86 & & & 10.04 & & & 23.26 & & \\
\hline Total & 179 & & & & & & & & & & & & \\
\hline Cochran's $C$ test & & 0.0849 & NS & & 0.0125 & NS & & 0.0976 & NS & & 0.1155 & NS & \\
\hline Transformation & & None & & & None & & & None & & & None & & \\
\hline SNK test for factor habitat & & & & & Islands & oasts $=b c$ & & & & & & & \\
\hline Mean $( \pm$ SE $)$ & & & & & $\begin{array}{r}6.47( \pm \\
\quad( \pm 0 .\end{array}$ & $\begin{array}{l}\text { 1) }>5.14 \\
=5.33( \pm\end{array}$ & & & & & & & \\
\hline
\end{tabular}

$N S$ not significant

$* P<0.05, * * P<0.01, * * * P<0.001$ 